ARTICLE

\title{
Unconventional molecule-resolved current rectification in diamondoid-fullerene hybrids
}

Jason C. Randel ${ }^{1,2}$, Francis C. Niestemski ${ }^{1,3}$, Andrés R. Botello-Mendez ${ }^{4}$, Warren Mar ${ }^{5}$, Georges Ndabashimiye ${ }^{1,2}$, Sorin Melinte ${ }^{6}$, Jeremy E.P. Dahl1, Robert M.K. Carlson', Ekaterina D. Butova7, Andrey A. Fokin 7,8,

Peter R. Schreiner ${ }^{7}$, Jean-Christophe Charlier $^{4} \&$ Hari C. Manoharan 1,3

The unimolecular rectifier is a fundamental building block of molecular electronics. Rectification in single molecules can arise from electron transfer between molecular orbitals displaying asymmetric spatial charge distributions, akin to $p-n$ junction diodes in semiconductors. Here we report a novel all-hydrocarbon molecular rectifier consisting of a diamantane- $\mathrm{C}_{60}$ conjugate. By linking both $s p^{3}$ (diamondoid) and $s p^{2}$ (fullerene) carbon allotropes, this hybrid molecule opposingly pairs negative and positive electron affinities. The single-molecule conductances of self-assembled domains on Au(111), probed by lowtemperature scanning tunnelling microscopy and spectroscopy, reveal a large rectifying response of the molecular constructs. This specific electronic behaviour is postulated to originate from the electrostatic repulsion of diamantane- $C_{60}$ molecules due to positively charged terminal hydrogen atoms on the diamondoid interacting with the top electrode (scanning tip) at various bias voltages. Density functional theory computations scrutinize the electronic and vibrational spectroscopic fingerprints of this unique molecular structure and corroborate the unconventional rectification mechanism.

\footnotetext{
${ }^{1}$ SLAC National Accelerator Laboratory, Stanford Institute for Materials and Energy Sciences, Menlo Park, California 94025, USA. ${ }^{2}$ Department of Applied Physics, Stanford University, Stanford, California 94305, USA. ${ }^{3}$ Department of Physics, Stanford University, Stanford, California 94305, USA. 4 Institute of Condensed Matter and Nanosciences, Université catholique de Louvain, B-1348 Louvain-La-Neuve, Belgium. ${ }^{5}$ Department of Electrical Engineering, Stanford University, Stanford, California 94305, USA. ${ }^{6}$ Institute of Information and Communication Technologies, Electronics and Applied Mathematics, Université catholique de Louvain, B-1348 Louvain-La-Neuve, Belgium. ${ }^{7}$ Institute of Organic Chemistry, Justus-Liebig University, Heinrich-Buff-Ring 58, 35392 Giessen, Germany. ${ }^{8}$ Department of Organic Chemistry, Kiev Polytechnic Institute, UA-03056 Kiev, Ukraine. Correspondence and requests for materials should be addressed to H.C.M. (email: manoharan@stanford.edu).
} 


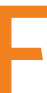
ollowing their discovery about a quarter-century ago $^{1}$, fullerenes attracted considerable attention for their fascinating geometry and have since become emblematic elements in the fabrication of complex ensembles of organized nanostructures $^{2-5}$. Their electronic properties, ranging from semiconductivity to superconductivity 6,7 , have spurred the search for derivatives encoding information stored in the arrangement of suitable binding sites, and for switching and reading out this information through interactions. Despite intensive efforts $^{8-10}$, the integration of fullerenes with other carbon allotropic forms and the identification of dynamic features for the controlled electromechanical switching between different superstructural arrangements remains considerably challenging.

Diamondoids $^{11,12}$ have intrinsic capabilities of self-assembly and electron transfer and therefore represent attractive candidates for building nanostructured materials, such as monochromatic high-efficiency field emitters ${ }^{13}$ or robust mechanical coatings. Additional features-including molecular geometries, selective functionalization and stacking interactions-enable the emergence of singular electronic phenomena in topologically favoured architectures ${ }^{14,15}$.

The development of large-area molecular devices has been established as an important area in nanoscience and nanotechnology applications, merging bottom-up self-assembly techniques with massively parallel architectures and fault-tolerant algorithms ${ }^{16}$. Fostered by pioneering work of Aviram and Ratner ${ }^{17}$, rectification in single molecules typically involves electron transfer between molecular orbitals displaying asymmetric probability amplitudes. This general mechanism has been applied to understanding rectification arising from electron tunnelling through molecular ${ }^{18}$ and supramolecular components ${ }^{19}$.

Here, we demonstrate that diamondoid properties can be readily transcribed into molecular rectifiers by their advantageous coupling with fullerenes via synthetic techniques. Accordingly, we probed by low-temperature scanning tunnelling microscopy
(STM) and spectroscopy (STS) the morphology and conductance of various self-assembled diamantane- $\mathrm{C}_{60}$ domains deposited at room temperature on $\mathrm{Au}(111)$ from diluted toluene solutions. Our data reveal an intriguing rectifying behaviour within the molecular constructs. This unconventional electronic behaviour likely originates from the electrostatic repulsion of Dia- $\mathrm{C}_{60}$ molecules due to the interaction of positively charged terminal hydrogen atoms on the diamondoid with the scanning tip at various bias voltages, in agreement with charge density values estimated on the Dia- $\mathrm{C}_{60}$ hybrid molecule by density functional theory (DFT) calculations.

\section{Results}

Design and synthesis of the diamondoid-fullerene molecule. In an effort to design novel rectification-capable molecules, the diamantane-fullerene combination was selected because of the optimal energy level configurations displayed by these two carbon structures (Supplementary Fig. 1). More specifically, the lowest unoccupied molecular orbital (LUMO) of $\mathrm{C}_{60}$ lines up closer to the Fermi level of the metallic substrates than does its highest occupied molecular orbital (HOMO). Seminal optical absorption measurements ${ }^{20}$ revealed that the opposite holds for diamantane. This suggests that diamantane- $\mathrm{C}_{60}\left(\mathrm{Dia}-\mathrm{C}_{60}\right)$ hybrid molecules should be ideal molecular rectifiers by combining a negative electron affinity moiety (localized on diamantane) with a positive electron affinity segment (localized on $\mathrm{C}_{60}$ ). Since $\mathrm{C}_{60}$-noble metal electrode interfaces exhibit reduced variability in transport characteristics $^{21}$, gold substrates are a suitable choice for examining the electronic performance of the physisorbed Dia$\mathrm{C}_{60}$ conjugate.

The 2-(diamant-4-yl)butadiene-1,3- $\mathrm{C}_{60}$ adduct $\left(\mathrm{Dia}-\mathrm{C}_{60}\right)$ was synthesized through thermal Diels-Alder reaction of 4-butadienyl diamantane and $\mathrm{C}_{60}$ in $84 \%$ yield ${ }^{22}$. The DFT-computed average orbital density for the isolated Dia- $\mathrm{C}_{60}$ construct along the molecular axis versus energy is shown in Fig. 1a (see also the
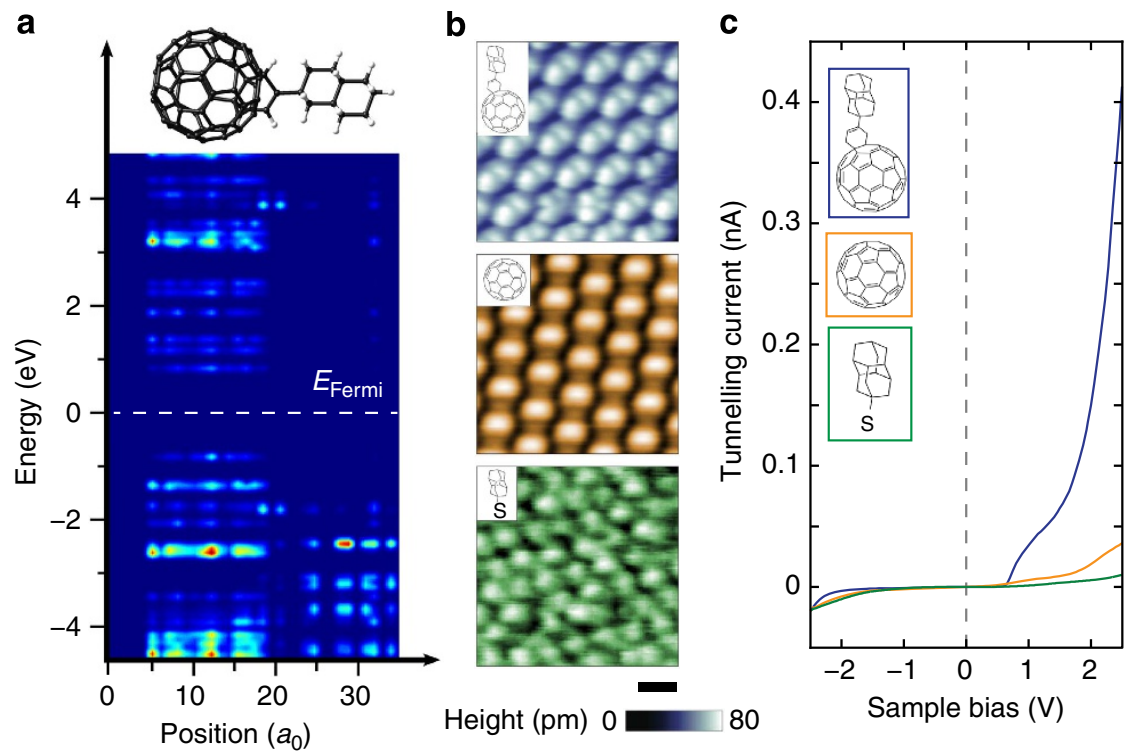

Figure 1 | Energy diagram of Dia- $\mathbf{C}_{60}$ and comparison with constituent molecules. (a) Colour map of the projected density of states of the Dia- $\mathrm{C}_{60}$ molecule integrated over the $x-y$ plane as a function of the $z$-coordinate (molecular axis, in units of the Bohr radius $a_{0}$ ) and energy. The Fermi energy of the $\mathrm{Dia}-\mathrm{C}_{60}$ on $\mathrm{Au}(111)$ system is indicated by the dashed white line. To account for the HOMO-LUMO energy underestimation within DFT, a scissors operator (rigid shift) has been applied to correct the HOMO-LUMO separation. (b) $5 \times 5 \mathrm{~nm}^{2} \mathrm{STM}$ images of Dia- $\mathrm{C}_{60}\left(I=20 \mathrm{pA}, \mathrm{V}=2.5 \mathrm{~V}\right.$, top row), $\mathrm{C}_{60}$ $(I=200 \mathrm{pA}, V=2.5 \mathrm{~V}$, middle row) and diamantane-4-thiol $(I=20 \mathrm{pA}, V=2.0 \mathrm{~V}$, bottom row) covering Au(111) substrates. Scale bar, $1 \mathrm{~nm}$. (c) Currentvoltage spectra measured on the adlayers illustrated in $\mathbf{b}$. Tunnelling conditions for all measurements are $I=20 \mathrm{pA}$ and $V=-2.5 \mathrm{~V}$. Each curve corresponds to the average of a dozen of individual $I-V$ traces measured over the centres of various molecules in the adlayers. 
Methods Section and Suplementary Information, Supplementary Fig. 1). The resulting integrated projected density of states plot reveals the segregation of both diamantane- and $\mathrm{C}_{60}$-like energy levels on the two opposite ends of the molecule axis, and a negligible overlap across the cyclohexene linker. While this is an essential requirement for efficient molecular rectifiers, the energy levels do not simply conform to the donor-acceptor convention proposed by Aviram and Ratner ${ }^{17}$, namely, the $\mathrm{C}_{60}$-like states (acceptor states) lie entirely within the band-gap of the diamondoid-like states (donor states), rather than being offset from them. Hence, we do not expect the Dia- $\mathrm{C}_{60}$ molecules to behave as conventional Aviram-Ratner rectifiers.

Rectification and contrast with molecular constituents. The prominent submolecular features expressed by the Dia- $\mathrm{C}_{60}$ molecular arrays on the $\mathrm{Au}(111)$ surface are highlighted in Fig. $1 \mathrm{~b}$, in comparison with the hexagonal molecular arrangement of bare $\mathrm{C}_{60}$, and the denser diamantane-thiol self-assembled monolayers (SAMs) on gold substrates. Careful STM and STS studies of these SAM systems prove that the electronic behaviour of the Dia- $\mathrm{C}_{60}$ hybrid molecule is more complex than the sum of its constituents. Figure 1c presents the asymmetric electrical current $(I)$ versus bias voltage $(V)$ response of Dia- $C_{60}$, contrasting sharply with the recorded spectroscopic $I-V$ characteristics for the individual $\mathrm{C}_{60}$ and diamantane-4-thiol species.

Topographical structure of molecular monolayers. A longrange topograph spanning multiple $\mathrm{Dia}-\mathrm{C}_{60}$ domains is presented in Fig. 2a. An ordered two-dimensional lattice of Dia- $\mathrm{C}_{60}$ molecules is visible, as well as a seemingly disordered low-conductance region (dark-blue areas). Point-like defects consisting of missing single or few molecules are common, and affect $20 \pm 4 \%$ of the lattice sites. This molecular arrangement differs from the regular $\mathrm{C}_{60}$ SAM (inset to Fig. 2a), which essentially displays full coverage. Despite this decrease in coverage, the estimated lattice constant of $9.5 \pm 0.3 \AA$ for the $\mathrm{Dia}-\mathrm{C}_{60}$ SAM is close to the measured $\mathrm{C}_{60}$ spacing of $9.8 \pm 0.2 \AA$, both of which corroborate the $\approx 1.0 \mathrm{~nm}$ value reported in the literature ${ }^{3,23}$. Thus, we infer that the diamantane moiety is nearly vertically oriented in Dia$\mathrm{C}_{60}$ adsorbed on $\mathrm{Au}(111)$ substrates, suggesting a generic hosting property of other metallic surfaces for Dia- $\mathrm{C}_{60}$ molecules. The low coverage of Dia- $\mathrm{C}_{60}$ indicates that the presence of the diamondoid alters the delicate equilibrium between the fullerenesurface and molecule-molecule interactions responsible for producing high-coverage SAMs. We attribute the lowconductance regions to disordered, prostrate Dia- $\mathrm{C}_{60}$ molecules (or fragments thereof), or solvent molecules that are not displaced by the Dia- $\mathrm{C}_{60}$ during SAM formation. In some areas, the lowconductance regions exhibit self-assembled domains of toluene molecules (see insets to Fig. 2b). This is likely a byproduct of the long-range physisorption of the Dia- $\mathrm{C}_{60}$ dyads on gold surfaces, further evidencing the fact that the diamondoid-fullerene lattice is less stable than that of $\mathrm{C}_{60}$, for which no self-organized toluene or low-conductance regions were detected. The possiblity that the toluene layer exists underneath the Dia- $\mathrm{C}_{60}$ lattice can be ruled out for a number of reasons. If the molecular arrangement of Dia- $\mathrm{C}_{60}$ on toluene was stable on $\mathrm{Au}(111)$, we would expect at least a coverage-dependent formation of different toluene and paired Dia- $\mathrm{C}_{60}$ on toluene domains for various self-assembly times. Instead, increasing the exposure time of the gold substrates to the molecular solution negligibly improves the overall Dia- $\mathrm{C}_{60}$ coverage. Second, we would predict some of the isolated Dia- $\mathrm{C}_{60}$ molecules lying on toluene-which can easily be identified spectroscopically-to have the same height as those in the Dia- $\mathrm{C}_{60}$ lattice, which we do not observe (Fig. 2b). Finally,

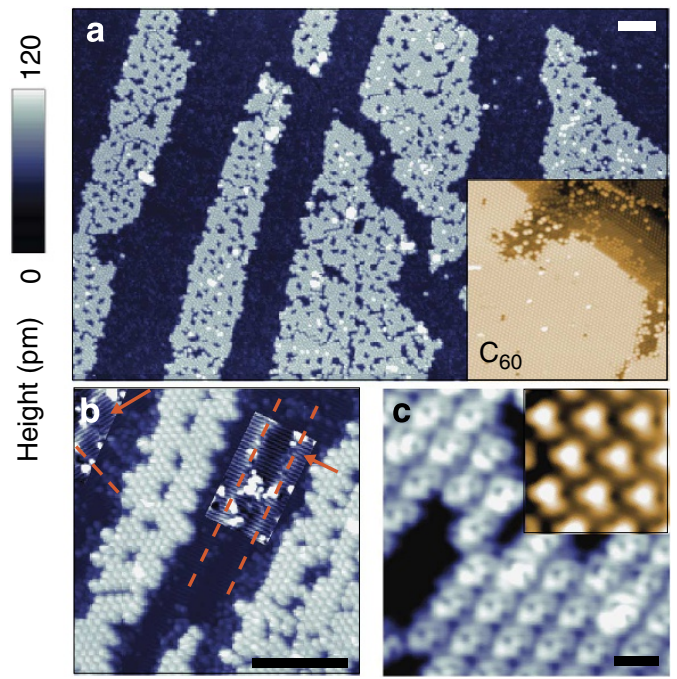

Figure 2 | STM images displaying the packing and structure of Dia- $\mathrm{C}_{60}$ adlayers. (a) Long-range $160 \times 100 \mathrm{~nm}^{2}$ STM image of hexagonally packed Dia- $C_{60}$ molecules on $\mathrm{Au}(111)$ with $42 \pm 3 \%$ coverage $(I=20 \mathrm{pA}$, $V=2.5 \mathrm{~V}$ ). Inset: for reference, $\mathrm{C}_{60}$ hexagonally packed on $\mathrm{Au}(111)$ with nearly $100 \%$ coverage $(I=100 \mathrm{pA}, V=2.0 \mathrm{~V})$. Same scale as in the Dia- $C_{60}$ topography image a. Scale bar, $10 \mathrm{~nm}$. (b) A $30 \times 30-\mathrm{nm}^{2}$ topography image of $\mathrm{Dia}-\mathrm{C}_{60}$ molecules on $\mathrm{Au}(111)$. Dark regions outside the two domains are covered by toluene molecules organized in stripes. Contrast in boxed regions is enhanced to make toluene stripes (orange arrows) and the underlying $\mathrm{Au}$ (111) herringbone reconstruction (dashed orange lines) visible. Scale bar, $10 \mathrm{~nm}$. (c) A $6.5 \times 6.5-\mathrm{nm}^{2}$ topography image of Dia- $\mathrm{C}_{60}$ molecules on $\mathrm{Au}(111)$ showing the submolecular structure $(I=20 \mathrm{pA}, V=-2.5 \mathrm{~V})$. Inset: zoom into the $C_{60}$ adlayer $(I=100 \mathrm{pA}$, $V=-2.5 \mathrm{~V}$ ). Inset is same scale as main image c. Scale bar, $1 \mathrm{~nm}$. At this particular bias voltage, $\mathrm{C}_{60}$ molecules exhibit threefold symmetry, whereas Dia- $\mathrm{C}_{60}$ dyads do not.

neighbouring strips of toluene rows caused by the $\mathrm{Au}(111)$ herringbone reconstruction are offset from one another by half the width of a toluene row, whereas the Dia- $\mathrm{C}_{60}$ lattice extends without defect over the length scale of the herringbone pattern.

High-resolution topographs over Dia- $\mathrm{C}_{60}$ and $\mathrm{C}_{60}$ molecules reveal bias-dependent submolecular-scale features (Fig. 2c). The well-known threefold and twofold symmetry patterns-visible in the $\mathrm{C}_{60}$ SAMs on $\mathrm{Au}(111)$ at negative and positive $V$, respectively-are reduced in the Dia- $\mathrm{C}_{60}$ SAM because of the diamondoid. Indeed, the effect of the diamondoid's presence on the topographic appearance of the fullerene-derivative adlayer is especially perceptible at $V=-2.5 \mathrm{~V}$ (Fig. 2c, lower left), where a protrusion is visible in the lower left of each molecule. This eccentric asymmetry matches the calculated projected density of states (Supplementary Fig. 1), since the dominant contribution of the diamondoid in the electronic states is found to occur at approximately $-2.0 \mathrm{eV}$ below the Fermi level. The unoccupied states $(V>0)$ present minor variations compared with the $\mathrm{C}_{60}$ states, both exhibiting a two-lobed shape, although a more submolecular structure can be observed on the Dia- $\mathrm{C}_{60}$ topographs (that is, lower symmetry), presumably due to the lifted degeneracy caused by the chemical bonding of the diamondoid (Supplementary Figs 1-3).

Electronic spectroscopy of single molecules. We now turn to the electronic properties of the individual Dia- $\mathrm{C}_{60}$ molecules. The $I-V$ curve and the $d I / d V$ spectrum recorded over the centre of a Dia- $\mathrm{C}_{60}$ molecule are shown in Fig. 3a,b, along with typical $\mathrm{C}_{60}$ 


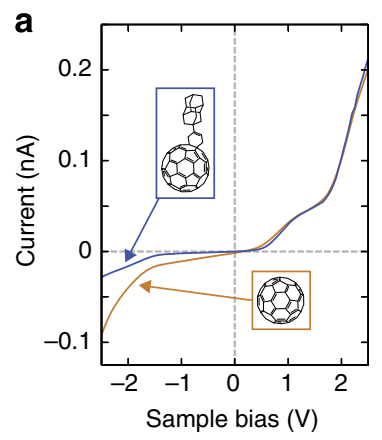

C

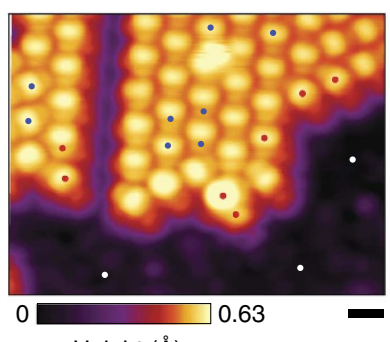

Height $(\AA)$

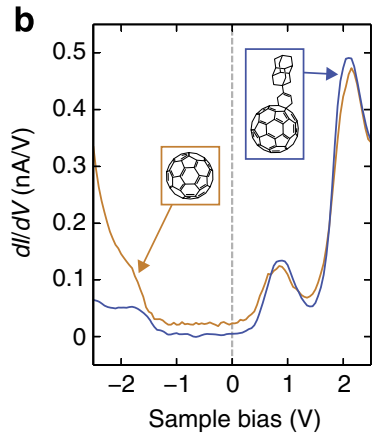

d

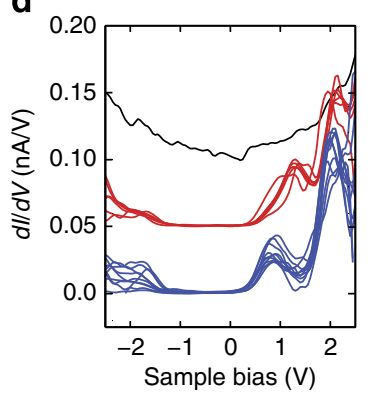

e

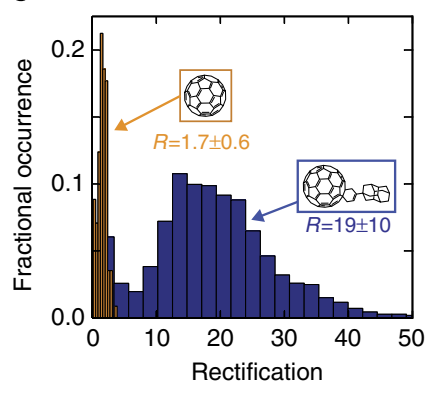

f

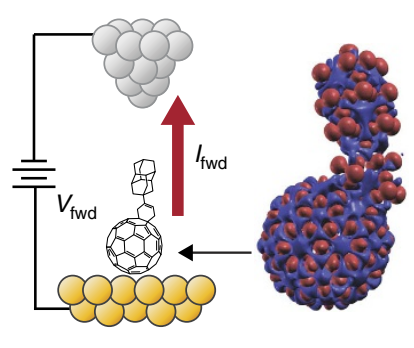

Figure 3 | Single-molecule $\mathbf{I}-\boldsymbol{V}$ and $\boldsymbol{d} \mathbf{I} / \boldsymbol{d} \boldsymbol{V}$ measurements and histogram of rectification factors at $\mathbf{4 . 5} \mathbf{K}$. (a) $I-V$ measurements over $D i a-C_{60}$ (blue) and $\mathrm{C}_{60}$ (orange) molecules highlighting their spectral similarity at positive bias and the suppression of conductance at negative bias for the Dia- $\mathrm{C}_{60}$ dyad. (b) $d l / d V$ point-spectra over the Dia- $C_{60}$ molecule (blue) and the $C_{60}$ molecule (orange) collected simultaneously with the $I-V$ curves shown in a. (c) Region with $\mathrm{Dia}-\mathrm{C}_{60}$ molecules over which the spectra shown in $\mathbf{d}$ were recorded. Dots in the adlayer represent the location of individual point-spectra, with colours corresponding to the $d l / d V$ spectra colours: blue refers to the middle of the lattice (six nearest neighbours) and red refers to the edge of the lattice ( $<6$ nearest neighbours). Scale bar, $1 \mathrm{~nm}$. (d) For reference, the black trace is the average of the spectra taken over the white dots in $\mathbf{c}$, located in a disordered sample area, and it has been shifted for clarity. The tunnelling junction was stabilized at $V=2.5 \mathrm{~V}$ before turning the feedback off and performing the measurements: $I=200 \mathrm{pA}(\mathbf{a}, \mathbf{b})$ and $100 \mathrm{pA}$ in $\mathbf{d}$. (e) Distribution of the rectification factors determined at $\pm 2.5 \mathrm{~V}$ for Dia- $\mathrm{C}_{60}$ (blue) and $\mathrm{C}_{60}$ (orange). (f) Sketch shows the orientation of the Dia- $\mathrm{C}_{60}$ on the gold surface and the direction of forward bias. Electronic charge density differences in an isolated Dia- $\mathrm{C}_{60}$ molecule where subtraction of each atomic contribution has been applied. This electronic density distribution provides information regarding the chemical behaviour of the Dia- $\mathrm{C}_{60}$ dyad, where the negative charge (blue) is more spatially localized on the $\mathrm{C}_{60}$ while the positive charge (red) is predicted closer to the diamantane moiety. Positive and negative electronic density isosurfaces correspond to \pm 0.05 electron $\AA^{-3}$, respectively.

measurements. In these measurements, the bias voltage has been set to $V=+2.5 \mathrm{~V}$ (compared with $-2.5 \mathrm{~V}$ in Fig. 1a) to prevent saturation of the lock-in detector used to measure $d I / d V$; the rectification is therefore manifested in these measurements as a decrease in the Dia- $\mathrm{C}_{60}$ current (relative to $\mathrm{C}_{60}$ ) for $V<0$, rather than an increase in current for $V>0$. Similarities are readily apparent between the STS spectra of Dia- $\mathrm{C}_{60}$ and $\mathrm{C}_{60}$, namely, local maxima in $d I / d V$ traces at voltages corresponding to the energies of the bare $\mathrm{C}_{60}$ HOMO, LUMO and LUMO + 1 states (compare with Supplementary Fig. 1a,c). The amplitude of the Dia- $\mathrm{C}_{60}$ conductance in the occupied states $(V<0)$ is, however, noticeably reduced compared with $\mathrm{C}_{60}$. Interestingly, the strongly asymmetric current in the Dia- $\mathrm{C}_{60}$ molecule arises in the opposite bias direction than the Aviram-Ratner effect predicts. For the particular $I-V$ curve discussed here, the Dia- $\mathrm{C}_{60}$ allows the passage of $\sim 6.5$ times more current at $2.5 \mathrm{~V}$ than at $-2.5 \mathrm{~V}$, while the $\mathrm{C}_{60}$ displays a rectification ratio of only 2.2 (where rectification ratio $R$ is defined as $|I(V) / I(-V)|$ at $V=2.5 \mathrm{~V})$. We find the conductance suppression at negative $V$-and the resulting asymmetric $I-V$ curve-to be the norm across many distinctive molecules, domains and samples. The resulting rectification behaviour reflects the qualitatively equivalent current-voltage data taken during spectroscopy in both positive to negative and reverse bias sweep directions (Figs 1 and 3). Its emergence alludes to the subtle role the diamondoid plays by contributing to the unoccupied molecular states of Dia- $\mathrm{C}_{60}$, which are located in close proximity of the Fermi level and, spatially, near the tip (see Fig. 1a). Figure 3c,d illustrates how STS measurements are observed to vary between different individual molecules. On one hand, the blue curves in Fig. 3d were all measured over molecules with six nearest neighbours, and all exhibit $\mathrm{C}_{60}$-like $\mathrm{HOMO}$, LUMO and LUMO + 1 peaks. On the other hand, STS measurements on molecules occupying the edge of the two-dimensional lattice (red curves in Fig. 3d) present an increase in the energy of the $\mathrm{C}_{60}$-like LUMO peak of $\sim 0.3 \mathrm{eV}$. This effect has been previously observed in $\mathrm{C}_{60}$ adlayers ${ }^{24}$, and has been attributed to differences in the intramolecular screening occurring at the edge of the lattice. The fact that a similar effect is observed in our diamantane-fullerene samples points out that the intramolecular interactions seen in $\mathrm{C}_{60}$ adlayers are likewise present in Dia- $\mathrm{C}_{60}$ lattices.

The rectification ratio has been determined for $\sim 10^{3}$ individual $I-V$ measurements recorded over Dia- $\mathrm{C}_{60}$ molecules. A histogram of these results is shown in Fig. 3e together with a histogram computed from 200 measurements taken on a $\mathrm{C}_{60}$ monolayer. The particular electronic structure of the Dia- $\mathrm{C}_{60}$ molecule enables one order of magnitude higher average rectification. Reports of unimolecular rectification in the literature vary widely, spanning a range from slightly greater than unity to higher than 1,000, and are typically reported for voltages up to $3 \mathrm{~V}$ (ref. 18). These values come from single-molecule measurements in STM or break-junction geometries as well as measurements of thin layers with conducting atomic force microscopy probes and large-area devices with molecular tunnelling junctions ${ }^{19}$. 

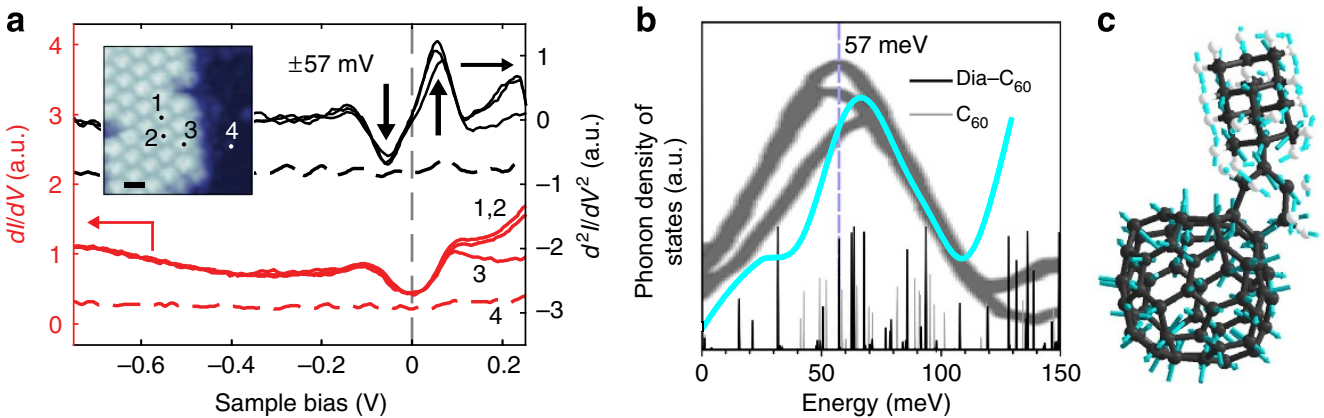

Figure 4 | Low-voltage inelastic tunnelling spectroscopy and computed dynamical properties in Dia-C $\mathbf{C}_{\mathbf{6 0}}$. (a) $d l / d V\left(\mathrm{red}, \mathrm{left}\right.$ axis) and $d^{2} I / d V^{2}$ (black, right axis) measurements of Dia- $\mathrm{C}_{60}$ molecules in an adlayer and the adjacent disorder area $(I=100 \mathrm{pA}, \mathrm{V}=-0.75 \mathrm{~V})$ at $4.5 \mathrm{~K}$. The curves were recorded at the locations indicated in the inset. Scale bar, $1 \mathrm{~nm}$. Solid curves (1-3) were measured over the Dia- $\mathrm{C}_{60}$ molecules and display anti-symmetric peaks at $\pm 57 \mathrm{meV}$, whereas the dashed trace (4) was collected over the disordered region. (b) Phonon density of states of Dia- $\mathrm{C}_{60}$ (black spectrum) and $\mathrm{C}_{60}$ (light-grey spectrum) molecules. The background image is the $d^{2} l / d V^{2}$ curve already illustrated in $\mathbf{a}$. The cyan curve is the computed Dia- $\mathrm{C}_{60}$ phonon spectrum with a $20 \mathrm{meV}$ broadening chosen to match the experimentally observed linewidths. The dashed line indicates the centre of the $d^{2} I / d V^{2}$ peak at $57 \mathrm{meV}$, which corresponds to a phonon mode illustrated in $\mathbf{c}$ by the arrows in the ball-and-stick Dia- $\mathrm{C}_{60}$ model. Most phonon modes within the $50-70 \mathrm{meV}$ range involve vibrations of the whole molecule. (c) The $57 \mathrm{meV}$ vibrational mode of the hybrid molecule is displayed with the ball-and-stick Dia- $\mathrm{C}_{60}$ model.

The Dia- $\mathrm{C}_{60}$ molecule represents to our knowledge the first molecular rectifier that is a pure hydrocarbon, pairs two molecules displaying negative and positive electron affinities, respectively, and combines a caged $s p^{3}$ molecule with a spherical $s p^{2}$ network.

Vibrational spectroscopy of single molecules. Additional insight into the transport mechanisms responsible for unimolecular rectification can be obtained via vibronic spectroscopy ${ }^{14,25-28}$. The presence of inelastic modes in molecule-adsorbed metallic surfaces can be detected as anti-symmetric peaks in $d^{2} I / d V^{2}$ spectra $^{29}$. Low-bias spectroscopy reveals a distinct signature of such modes over the Dia- $\mathrm{C}_{60}$ adlayer (Fig. 4a). The $d^{2} I / d V^{2}$ peak at $57 \mathrm{mV}$ is only present over the Dia- $\mathrm{C}_{60}$ molecules, indicating that we are exciting vibrational modes localized to the hybrid construct. To understand this spectroscopic feature, the vibrational spectrum of the Dia- $\mathrm{C}_{60}$ molecule has been computed using DFT and compared with the $\mathrm{C}_{60}$ spectrum in the corresponding energy range. A series of low-frequency modes in the vicinity of $57 \mathrm{meV}$ can be observed in Fig. $4 \mathrm{~b}$, resulting in a peak in the phonon spectrum closely matching the spectroscopy data. Most of these vibrational modes involve all the atomic constituents of the Dia- $\mathrm{C}_{60}$ hybrid, although a few of them correspond to localized atomic motions in the diamantane structure (Fig. 4c; Supplementary Fig. 4). Indeed, $\mathrm{Li}$ et al. ${ }^{30}$ recently highlighted the softening of the vibrational modes at metal- $\mathrm{C}_{60}$ interfaces due to both external electric fields, and doping or charge transfer. Similarly, the $\mathrm{C}_{60}$-dia molecule exhibiting a covalent binding between the two components reveals at low energies a strong coupling between the respective vibration modes. The DFT computations provide further evidence for the diamondoid-up structural orientation, as tunnelling electrons should couple most strongly to vibrational modes located on the part of the molecule closest to the tip.

\section{Discussion}

The unique structure of the diamantane-fullerene dyad potentially facilitates an unconventional operation for the molecular rectifier. This rectification mechanism relies on the presence of the hydrogen atoms at the diamondoid surface that are partially positively charged, as illustrated in Fig. $3 \mathrm{f}$. When a positively biased tip $(V<0)$ is brought close to the tip of the molecule, we expect the positively charged hydrogen atoms to be repelled by the tip. Accordingly, the top part of the molecule rotates away from the tip. In the case of STM measurements, this effect would cause the current to decrease (due to increased tip-molecule distance) whenever the tip bias becomes more positive at constant height-as in $d I / d V$ spectroscopy. As a result, the current would be reduced when the sample bias sweeps from positive to negative, producing a rectifying effect consistent with the observed behaviour shown in Fig. 3a. This type of molecular motion could be related to the inelastic tunnelling signature observed in Fig. 4, especially since it is suggestive of a high degree of motion of the diamondoid. Indeed, this field-induced motion effect could be observed in these fullerene derivatives because of the subtle intramolecular dynamics of the hydrogen-terminated diamantane interacting with the tip electrode.

In summary, we demonstrate that the low-temperature $I-V$ characteristics of a diamondoid-fullerene hybrid molecule, probed by scanning tunnelling spectroscopy, are strikingly different than those of its constituents. The Dia- $\mathrm{C}_{60}$ dyads essentially display a $\mathrm{C}_{60}$-like spectrum in which tunnelling through the occupied states is suppressed, while the throughmolecule tunnelling process seems influenced by diamondoid molecular motions that remain unconstrained in the ordered assemblies. This work can be further exploited to design novel hydrocarbon-based single-molecule rectifiers.

\section{Methods}

Materials. Synthesis of 2-(diamant-4-yl)butadiene-1,3- $C_{60}$ adduct (Dia- $\left.\mathrm{C}_{60}\right)$ : a mixture of $0.1 \mathrm{~g}(0.140 \mathrm{mmol})$ of fullerene $\mathrm{C}_{60}$ and $34 \mathrm{mg}(0.140 \mathrm{mmol})$ of 2 -(diamant-4-yl)butadiene prepared as previously described ${ }^{22}$ was refluxed under argon in $100 \mathrm{ml}$ of dry toluene for $4.5 \mathrm{~h}$. Excess solvent was evapourated; the remainder was dissolved in $\mathrm{CS}_{2}$ mixed with $15 \mathrm{ml}$ of pentane and stirred at room temperature for $10 \mathrm{~min}$, filtered, washed with pentane, dried in vacuo and stored dry to give $112 \mathrm{mg}(84 \%)$ of a cherry-brown powder. ${ }^{1} \mathrm{H} \mathrm{NMR}\left(\delta, \mathrm{CDCl}_{3}\right): 6.40$ $(\mathrm{m} 1 \mathrm{H}), 3.84(\mathrm{~m} 4 \mathrm{H}), 1.82(\mathrm{~m} 8 \mathrm{H}), 1.65(\mathrm{~m} 11 \mathrm{H}) .{ }^{13} \mathrm{C} \mathrm{NMR}\left(\delta, \mathrm{CDCl}_{3}\right): 156.8(\mathrm{C})$, 145.6 (C), $145.4(\mathrm{C}), 144.7$ (C), 143.1 (C), 143.0 (C), 142.6 (C), 142.4 (C), 142.3 (C), $142.1(\mathrm{C}), 141.6(\mathrm{C}), 140.1(\mathrm{C}), 120.9(\mathrm{CH}), 66.2(\mathrm{C}), 66.0(\mathrm{C}), 42.2\left(\mathrm{CH}_{2}\right), 40.5$ $\left(\mathrm{CH}_{2}\right), 40.0\left(\mathrm{CH}_{2}\right), 38.2\left(\mathrm{CH}_{2}\right), 38.1\left(\mathrm{CH}_{2}\right), 37.2(\mathrm{CH}), 36.5(\mathrm{C}), 26.1(\mathrm{CH})$. Mass spectrometry: 960 (<1\%), 720 (100\%), 240 (45\%), 202 (100\%). High-resolution mass spectrometry: found 961.1632 , calculated for $\mathrm{C}_{78} \mathrm{H}_{24} 960.1878$.

STM experiments. $\mathrm{Au}(111)$ on mica samples (Agilent) were rinsed with acetone and isopropanol, mounted on a sample holder and transferred to the ultra-highvacuum preparation chamber. The sample is cleaned by repeated cycles of ion sputtering $(2 \mathrm{keV}, 15 \mathrm{~min})$ and annealing $\left(540^{\circ} \mathrm{C}, 45 \mathrm{~min}\right)$. It is then removed to 
the load-lock chamber under a $5-1 \mathrm{~min}^{-1}$ flow of nitrogen gas to minimize backflow of air towards it. A $\sim 100 \mu \mathrm{l}$ drop of $0.01 \mathrm{mM}$ Dia- $\mathrm{C}_{60}$ in toluene is deposited on the sample and allowed to spread for $60 \mathrm{~s}$. Before the load-lock chamber is resealed and pumped down, the drop is blown off using nitrogen gas, the sample is rinsed with isopropanol and again blown dry with nitrogen gas. The sample is moved into the STM chamber (pressure below $2 \times 10^{-11} \mathrm{mbar}$ ), and cooled to $4.5 \mathrm{~K}$ for observation. STM measurements were typically performed with $\mathrm{Pt} / \mathrm{Ir}$ (90\%/10\%) tips. Bias voltage always refers to the sample potential with respect to the tip. I-V spectroscopy is performed by moving the tip to a particular location, turning the feedback loop off and ramping the voltage between the desired values while the current is recorded. $d I / d V$ versus $V$ spectra were collected in a similar fashion and with a lock-in amplifier, with a modulation of few $\mathrm{mV}$ added to the bias voltage.

Computations. DFT computations were performed using the SIESTA code $^{31}$ with a double-zeta polarized basis set, GGA (PBEsol) functional and Troullier-Martins pseudopotentials. Atomic structures were allowed to relax until forces were reduced to within $0.05 \mathrm{eV} / \AA \AA$. An energy cutoff of $300 \mathrm{Ry}$ and a uniform $8 \times 8 \times 1 k$-point mesh were used during the computations. STM topographs (at bias voltage $V$ ) were generated by summing the electron density of each eigenstate with energy $E$ between $E_{\mathrm{F}}$ and $E_{\mathrm{F}}+\mathrm{e} V$. The vibration mode simulations were carried out using a finite difference approach, and an energy cutoff of 1,000 Ry for accurate force constant calculation.

\section{References}

1. Kroto, H., Heath, J. R., O’Brien, S. C., Curl, R. F. \& Smalley, R. E. C60: buckminsterfullerene. Nature 318, 162-163 (1985).

2. Bonifazi, D. et al. Supramolecular nanostructuring of silver surfaces via self-assembly of [60]fullerene and porphyrin modules. Adv. Funct. Mater. 17, 1051-1062 (2007).

3. Schull, G. \& Berndt, R. Orientationally ordered $(7 \times 7)$ superstructure of C60 on $\mathrm{Au}(111)$. Phys. Rev. Lett. 99, 226105 (2007).

4. Otero, G. et al. Fullerenes from aromatic precursors by surface-catalysed cyclodehydrogenation. Nature 454, 865-868 (2008).

5. Wang, Y., Yamachika, R., Wachowiak, A., Grobis, M. \& Crommie, M. F. Tuning fulleride electronic structure and molecular ordering via variable layer index. Nat. Mater. 7, 194-197 (2008).

6. Park, H. et al. Nanomechanical oscillations in a single-C60 transistor. Nature 407, 57-60 (2000).

7. Winkelmann, C. B., Roch, N., Wernsdorfer, W., Bouchiat, V. \& Balestro, F. Superconductivity in a single-C60 transistor. Nat. Phys. 5, 876-879 (2009).

8. Vostrowsky, O. \& Hirsch, A. Heterofullerenes. Chem. Rev. 106, 5191-5207 (2006).

9. Lu, J., Yeo, P. S. E., Gan, C. K., Wu, P. \& Loh, K. P. Transforming C60 molecules into graphene quantum dots. Nat. Nanotech. 6, 247-252 (2011).

10. Smith, B. W., Monthioux, M. \& Luzzi, D. E. Encapsulated C60 in carbon nanotubes. Nature 396, 323-324 (1998).

11. Dahl, J. E., Liu, S. G. \& Carlson, R. M. K. Isolation and structure of higher diamondoids, nanometer-sized diamond molecules. Science 299, 96-99 (2003).

12. Schwertfeger, H., Fokin, A. A. \& Schreiner, P. R. Diamonds are a chemist's best friend: diamondoid chemistry beyond adamantane. Angew. Chem. Int. Ed. 47, 1022-1036 (2008).

13. Yang, W. L. et al. Monochromatic electron photoemission from diamondoid monolayers. Science 316, 1460-1462 (2007).

14. Wang, Y. et al. Spatially resolved electronic and vibronic properties of single diamondoid molecules. Nat. Mater. 7, 38-42 (2008).

15. Dameron, A. A., Charles, L. F. \& Weiss, P. S. Structures and displacement of 1-adamantanethiol self-assembled monolayers on $\mathrm{Au}\{111\}$. J. Am. Chem. Soc. 127, 8697-8704 (2005).

16. Palma, C.-A. \& Samorì, P. Blueprinting macromolecular electronics. Nat. Chem. 3, 431-436 (2011).

17. Aviram, A. \& Ratner, M. A. Molecular rectifiers. Chem. Phys. Lett. 29, 277-283 (1974).

18. Heath, J. R. Molecular electronics. Annu. Rev. Mater. Res. 39, 1-23 (2009).

19. Wimbush, K. S. et al. Control over rectification in supramolecular tunneling junctions. Angew. Chem. Int. Ed. 49, 10176-10180 (2010).

20. Landt, L. et al. The influence of a single thiol group on the electronic and optical properties of the smallest diamondoid adamantane. J. Chem. Phys. 132, 024710-024716 (2010).

21. Martin, C. A. et al. Fullerene-based anchoring groups for molecular electronics. J. Am. Chem. Soc. 130, 13198-13100 (2008).
22. Fokin, A. A. et al. Simple preparation of diamondoid 1,3-dienes via oxetane ring opening. Org. Lett. 9, 2541-2544 (2007).

23. Altman, E. I. \& Colton, R. J. Determination of the orientation of C60 adsorbed on $\mathrm{Au}(111)$ and $\operatorname{Ag}(111)$. Phys. Rev. B 48, 18244-18249 (1993).

24. Torrente, I. F., Franke, K. J. \& Pascual, J. I. Spectroscopy of C60 single molecules: the role of screening on energy level alignment. J. Phys. Condens. Matter 20, 184001 (2008).

25. Porath, D., Levi, Y., Tarabiah, M. \& Millo, O. Tunneling spectroscopy of isolated $\mathrm{C} 60$ molecules in the presence of charging effects. Phys. Rev. B 56, 9829-9833 (1997).

26. Bruot, C., Hihath, J. \& Tao, N. Mechanically controlled molecular orbital alignment in single molecule junctions. Nat. Nanotech. 7, 35-40 (2012).

27. Gross, L. Recent advances in submolecular resolution with scanning probe microscopy. Nat. Chem. 3, 273-278 (2011).

28. Joachim, C., Gimzewski, J. K. \& Tang, H. Physical principles of the single-C60 transistor effect. Phys. Rev. B 58, 16407-16417 (1998).

29. Pradhan, N. A., Liu, N. \& Ho, W. Vibronic spectroscopy of single C60 molecules and monolayers with the STM. J. Phys. Chem. B 109, 8513-8518 (2005).

30. Li, Y., Doak, P., Kronik, L., Neaton, J. B. \& Natelson, D. Voltage tuning of vibrational mode energies in single-molecule junctions. Proc. Natl Acad. Sci. USA 111, 1282-1287 (2014).

31. Soler, J. M. et al. The SIESTA method for ab-initio order-N materials simulation. J. Phys. Condens. Matter. 14, 2745-2779 (2002).

\section{Acknowledgements}

This work was primarily supported by the US Department of Energy, Office of Science, Basic Energy Sciences, Materials Sciences and Engineering Division, under contract DE-AC02-76SF00515. Nanomaterials quantum calculations (W.M. and G.N.) were supported by the US National Science Foundation (DMR-1206916). F.C.N. acknowledges support from the Karl van Bibber Fellowship. W.M. acknowledges support from an NDSEG fellowship. A.R.B.-M. acknowledges support from the M. de Merre Prize of Louvain. This research connects to the F.R.S.-FNRS of Belgium and to the ARC projects entitled 'Graphene StressTronics' (No 11/16-037) and 'TINTIN' (No 09/14-023) sponsored by the Communauté Française de Belgique (A.R.B.-M., S.M., J.-C.C.), and computational resources for DFT were provided by the CISM of the Universite catholique de Louvain (GREEN and LEMAITRE computers). P.R.S. and E.D.B. were supported through the Deutsche Forschungsgemeinschaft (Germany) through a DFG-NSF grant (CHE-0822112). A.A.F. was supported by the Ministry of Science and Education of Ukraine. We thank W. Clay, T. Devereaux, J. Fabbri, N. Melosh, A. Sorini and Z. X. Shen for discussions.

\section{Author contributions}

J.E.P.D., R.M.K.C., E.D.B., A.A.F. and P.R.S. designed and synthesized the materials. S.M. and H.C.M. supervised the study at Stanford University and SLAC. J.C.R. and F.C.N. planned, executed and analysed experiments with additional feedback from calculations performed by W.M. and G.N. A.R.B.-M. and J.-C.C. developed the theoretical model and performed the ab initio simulations. J.C.R., A.R.B.-M., S.M., J.-C.C. and H.C.M. wrote the paper with input from all authors. J.C.R., A.R.B.-M. and S.M. contributed equally to this work.

\section{Additional information}

Supplementary Information accompanies this paper at http://www.nature.com/ naturecommunications

Competing financial interests: The authors declare no competing financial interests.

Reprints and permission information is available online at http://npg.nature.com/ reprintsandpermissions/

How to cite this article: Randel, J. C. et al. Unconventional molecule-resolved current rectification in diamondoid-fullerene hybrids. Nat. Commun. 5:4877 doi: $10.1038 /$ ncomms5877 (2014)

This work is licensed under a Creative Commons AttributionNonCommercial-NoDerivs 4.0 International License. The images or other third party material in this article are included in the article's Creative Commons license, unless indicated otherwise in the credit line; if the material is not included under the Creative Commons license, users will need to obtain permission from the license holder to reproduce the material. To view a copy of this license, visit http:// creativecommons.org/licenses/by-nc-nd/4.0/ 